\title{
Eccrine Poromatosis Following Lymphoma Treatment: A Case Report and Literature Review
}

\author{
Suzanne Alkul, MD¹, Rachel Graubard, BS ${ }^{1}$, Carina Wasko, MD¹ \\ ${ }^{1}$ Department of Dermatology, Baylor College of Medicine, Houston, TX
}

\section{ABSTRACT}

Eccrine poromas are benign tumors that arise from the eccrine sweat ducts, commonly presenting as solitary lesions. Eccrine poromatosis, the sudden eruption of multiple eccrine poromas, is a rare phenomenon that generally occurs in immunosuppressed patients at any time after receiving treatment for malignancy. We report a case of eccrine poromatosis in a 79-year-old male patient with a previous history of recurrent T-cell lymphoma. Over the course of his disease, he was treated with polychemotherapy, radiation, and a definitive bone marrow transplant. The patient presented to the dermatology clinic 18 years after his initial diagnosis with a new onset of pruritic papules on the neck and chest. Histologic evaluation revealed all lesions to be eccrine poromas. This is the longest reported time interval between initial diagnosis of a primary malignancy and development of eccrine poromatosis. There is no evidence at this time to suggest that appearance of such lesions is indicative of cancer recurrence; therefore, there is no indication for further oncoloqic evaluation.

\section{INTRODUCTION}

Eccrine poromas, benign adnexal neoplasms of the eccrine sweat ducts, arise gradually as isolated lesions on the palms or soles, where sweat glands are highly concentrated. Tumors manifest clinically as tender or asymptomatic, erythematous papules or nodules. ${ }^{1}$ On histological examination, eccrine poromas are characterized by a well-circumscribed, uniform proliferation of glycogen-rich cuboidal cells with abundant eosinophilic cytoplasm and scattered ducts, extending into the dermis. ${ }^{2}$ Rarely, patients present with a sudden eruption of multiple poromas, a phenomenon known as poromatosis. While the pathogenesis is unclear, cases of eccrine poromatosis are typically reported in immunocompromised patients who have received chemotherapy, radiation, or chronic immunosuppressive therapy following stem cell transplantation. ${ }^{3-19}$ Occurrence of lesions has also been attributed to genetic predisposition, radiation-induced damage, or human papillomavirus, all contributing to impaired immunity. ${ }^{4-8}$ Crops of lesions may appear shortly after the onset of therapy or years after completion. Herein, we present a case of eccrine poromatosis in a patient with a previous history of T-cell lymphoma, initially diagnosed 18 years prior to presentation and eventually achieving sustained remission after 4 years with a combination of chemotherapy, radiation, and salvage stem cell transplant.

\section{CASE PRESENTATION}

A 79-year-old Hispanic man presented with a several month history of 5 pruritic, domeshaped, erythematous papules on the left 


\section{SKIN}

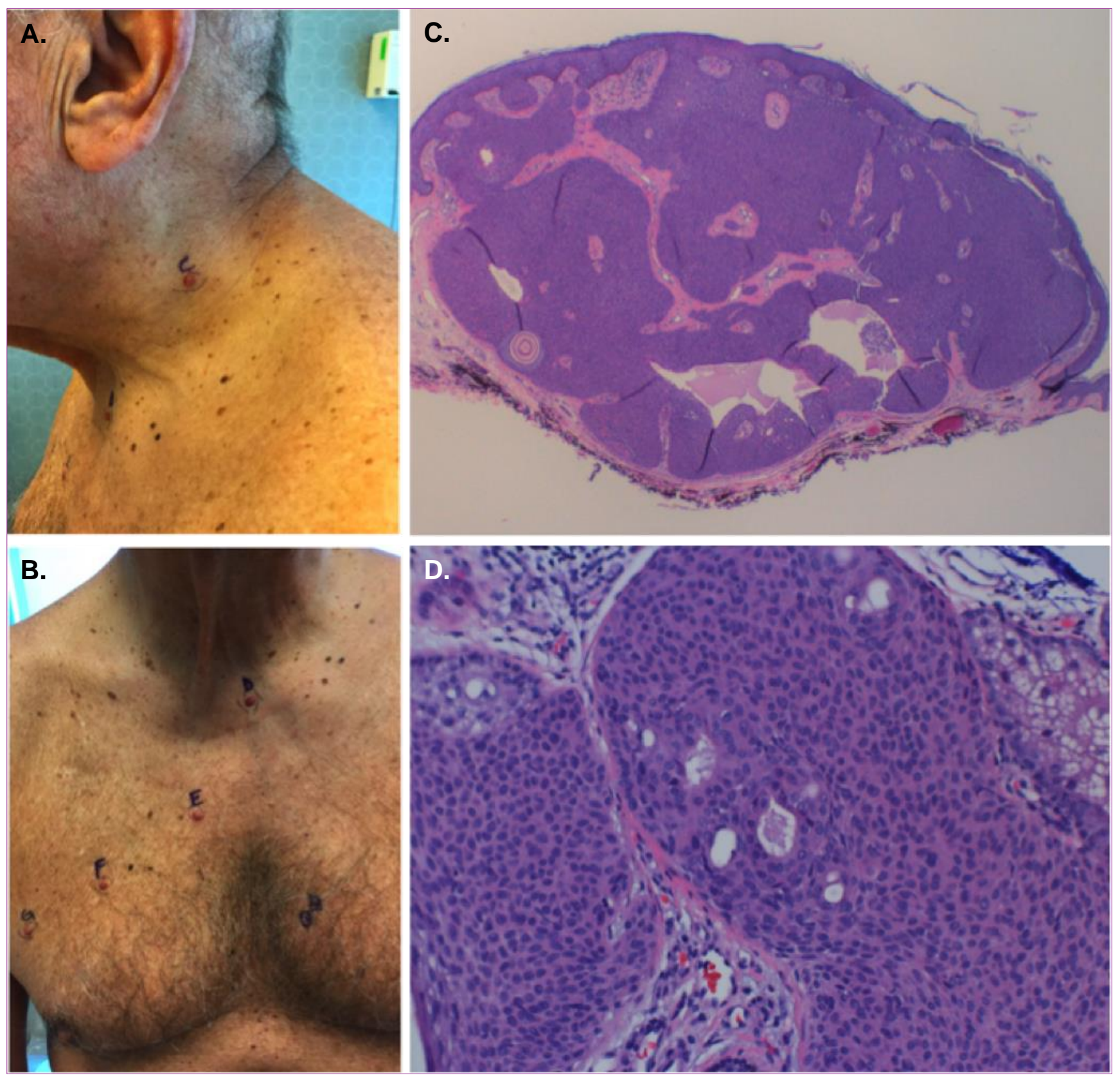

Figure 1. (A) Left neck with one erythematous papule. (B) Upper mid chest and right chest with 4 erythematous papules. (C) Broad anastomosing bands of monotonous epithelial cells with a broad connection to the epidermis. (H\&E, 20X). (D) Basophilic epithelial nuclei and sweat ducts within the tumor. (H\&E, 200x)

neck and right chest in a seatbelt distribution (Insert Figure 1). His previous medical history is significant for stage $1 \mathrm{~A}$ T-cell lymphoma, diagnosed 18 years prior to presentation, for which he received 6 cycles of cyclophosphamide, doxorubicin, vincristine, and prednisone (CHOP). $\mathrm{He}$ relapsed for the first time 3 years later and was treated with radiation therapy, achieving complete remission. After a second recurrence 1 year later, he received 3 additional cycles of etoposide, methylprednisolone, cytarabine, and cisplatin (ESHAP). This was followed by a 
sibling allogeneic stem cell transplant, for which he was conditioned with etoposide, prednisone, vincristine, cyclophosphamide, doxorubicin (EPOCH), and fludarabine. Post-transplant course was complicated by minor graft-versus-host disease (GVHD) of the skin. He has been in complete remission and has not required any immunosuppressive medications since then. All 5 lesions were treated with shave removal followed by electrodessication. Histology revealed thick anastomosing bands of monotonous epithelial cells, which had a broad connection to the epidermis and interspersed eccrine ducts (Figure 1). No further treatment was pursued. The patient has had no recurrence of the treated lesions nor development of any new poromas.

\section{DISCUSSION}

The simultaneous eruption of multiple eccrine poromas is an exceedingly rare phenomenon, and the exact etiology remains poorly understood. While the immunosuppressive effects of infection, pregnancy, and certain medications have been cited as potential catalysts for sudden development of multiple tumors, the first two reported patient cases of poromatosis did not include a previous history of immunosuppression or other predisposing risk factors. ${ }^{7,18,20-23}$ Other cases have been attributed to systemic lupus erythematosus, requiring chronic immunosuppressive therapy, or predisposing genetic conditions, such as hidrotic ectodermal dysplasia. ${ }^{24,25}$ Interestingly, in a canine patient, multiple eccrine poromas were found following amputation of a chronically inflamed paw, previously treated with laser surgery to correct a congenital anomaly. ${ }^{26}$ Because poromas are benign, adnexal tumors that arise from the sweat gland duct, they may originate from either eccrine or apocrine lineages. While eccrine poromas comprise the vast majority of reported cases, apocrine poromas occur with less frequency. Clinically, both lesions present similarly as singular, flesh-colored papules on the acral surfaces; therefore, lesions must be differentiated histologically. ${ }^{2}$ Unlike eccrine poromatosis, cases of multiple apocrine poromas have not been associated with a previous history of immunosuppression. ${ }^{27,28}$ Overall, reports of eccrine poromatosis have demonstrated a strong association with lymphoproliferative malignancies treated with immunosuppressive therapies; however, their clinical course is unpredictable, as lesions may appear at any time. 3-19 Presently, the longest reported period of time between onset of malignancy and emergence of eccrine poromatosis is 16 years. ${ }^{8}$ This patient presented 18 years after his original diagnosis with $\mathrm{T}$ cell lymphoma. To our knowledge, this is the longest interval between onset of a primary malignancy and emergence of eccrine poromatosis and the first case associated with non-cutaneous $T$ cell lymphoma.

Out of 21 reported cases of eccrine poromatosis associated with malignancy, 7 patients were primarily diagnosed with acute myeloid leukemia, and 10 patients were diagnosed with a particular subtype of nonHodgkin lymphoma (Table 1). Only one previously documented case has been associated with a neoplasm of T-cell origin. ${ }^{3}$ In the first case of malignancy-associated eccrine poromatosis, Kurokawa et al. (2001) described a 72 year-old male with mycosis fungoides, the most common form of cutaneous T-cell lymphoma. The patient was treated with multiple courses of electron beam radiation therapy for recurrence of skin lesions, and 6 years after his initial diagnosis, the first eccrine poroma was excised and diagnosed. Rather than a single eruption of multiple lesions, the patient 
Table 1. Reported Cases of Eccrine Poromatosis Associated with Malignancy

\begin{tabular}{|c|c|c|c|c|c|}
\hline Reference & $\begin{array}{l}\text { Patient Age \& } \\
\text { Gender }\end{array}$ & Associated Malignancy & Treatment Received & $\begin{array}{c}\text { Interval } \\
\text { between } \\
\text { diagnosis and } \\
\text { presentation }\end{array}$ & Comments \\
\hline $\begin{array}{l}\text { Kurokawa et al., 2001; } \\
\text { Myazaki, Japan }\end{array}$ & $\begin{array}{c}72 \\
\text { Male }\end{array}$ & Mycosis Fungoides & $\begin{array}{l}\text { Electron Beam } \\
\text { Radiation }\end{array}$ & 6 Years & \\
\hline $\begin{array}{l}\text { Mahlberg et al., 2006; } \\
\text { Philadelphia, } \mathrm{PA}^{4}\end{array}$ & $\begin{array}{c}42 \\
\text { Male }\end{array}$ & $\begin{array}{l}\text { Acute Lymphocytic } \\
\text { Leukemia (ALL) }\end{array}$ & $\begin{array}{l}\text { Chemotherapy; } \\
\text { Total Body Radiation; } \\
\text { Allogeneic Bone Marrow } \\
\text { Transplant }\end{array}$ & 2 Years & $\begin{array}{l}\text { Transplant complicated by } \\
\text { graft-versus-host disease } \\
\text { (GVHD) }\end{array}$ \\
\hline $\begin{array}{l}\text { Navi et al., 2008; } \\
\text { Davis, CA, }{ }^{5}\end{array}$ & $\begin{array}{c}64 \\
\text { Male }\end{array}$ & Non-Hodgkin Lymphoma & Chemotherapy & Unknown & \\
\hline $\begin{array}{l}\text { Sherman et al., 2010; } \\
\text { Oxford, United } \\
\text { Kingdom }^{6}\end{array}$ & $\begin{array}{l}32 \\
\text { Male }\end{array}$ & $\begin{array}{l}\text { Acute Myeloid Leukemia } \\
\text { (AML) }\end{array}$ & $\begin{array}{l}\text { Chemotherapy; } \\
\text { Total Body Radiation; } \\
\text { Allogeneic Bone Marrow } \\
\text { Transplant (2) }\end{array}$ & 6 Years & $\begin{array}{l}\text { Both transplants } \\
\text { complicated by GVHD }\end{array}$ \\
\hline $\begin{array}{l}\text { Diamantis et al., 2011; } \\
\text { Austin, } \text { TX }^{7}\end{array}$ & $\begin{array}{c}53 \\
\text { Male }\end{array}$ & Mantle Cell Lymphoma & $\begin{array}{l}\text { Allogeneic Stem Cell } \\
\text { Transplant }\end{array}$ & 5 Years & $\begin{array}{l}\text { Transplant complicated by } \\
\text { GVHD; } \\
\text { Lesions positive for HPV }\end{array}$ \\
\hline $\begin{array}{l}\text { Fujii et al., 2012; } \\
\text { Okayama, Japan8 }\end{array}$ & $\begin{array}{c}66 \\
\text { Female }\end{array}$ & $\begin{array}{l}\text { Chronic Lymphocytic } \\
\text { Leukemia (CLL); } \\
\text { B-Cell Follicular } \\
\text { Lymphoma }\end{array}$ & $\begin{array}{l}\text { Chemotherapy; } \\
\text { Radiation }\end{array}$ & 16 Years & \\
\hline $\begin{array}{l}\text { Fujii et al., 2012; } \\
\text { Okayama, Japan8 }\end{array}$ & $\begin{array}{c}62 \\
\text { Male }\end{array}$ & $\begin{array}{l}\text { Malignant Fibrous } \\
\text { Histiocytoma }\end{array}$ & $\begin{array}{l}\text { Chemotherapy; } \\
\text { Radiation }\end{array}$ & 10 Years & \\
\hline $\begin{array}{l}\text { Fujii et al., 2012; } \\
\text { Okayama, Japan }\end{array}$ & $\begin{array}{c}59 \\
\text { Male }\end{array}$ & B-Cell Lymphoma & Chemotherapy & 6 Months & \\
\hline $\begin{array}{l}\text { Fujii et al., 2012; } \\
\text { Okayama, Japan }\end{array}$ & $\begin{array}{c}72 \\
\text { Male }\end{array}$ & $\begin{array}{l}\text { B-Cell } \\
\text { Lymphoma }\end{array}$ & Chemotherapy & 10 Years & \\
\hline $\begin{array}{l}\text { Nguyen et al., } 2012 ; \\
\text { San Diego, } \text { CA }^{9}\end{array}$ & $\begin{array}{c}25 \\
\text { Male }\end{array}$ & AML & $\begin{array}{l}\text { Chemotherapy; } \\
\text { Autologous Bone } \\
\text { Marrow Transplant }\end{array}$ & 11 Years & \\
\hline
\end{tabular}




\section{SKIN}

\begin{tabular}{|c|c|c|c|c|c|}
\hline $\begin{array}{l}\text { Miura \& Yamamoto } \\
\text { 2013; } \\
\text { Fukushima, Japan }{ }^{10}\end{array}$ & $\begin{array}{c}72 \\
\text { Female }\end{array}$ & $\begin{array}{l}\text { Nasolacrimal Duct } \\
\text { Adenocarcinoma }\end{array}$ & Radiation & $\begin{array}{l}\text { Rapid onset } \\
\text { after radiation } \\
\text { therapy }\end{array}$ & \\
\hline $\begin{array}{l}\text { Garshick et al., 2014; } \\
\text { New York, NY11 }\end{array}$ & $\begin{array}{l}46 \\
\text { Male }\end{array}$ & AML & $\begin{array}{l}\text { Chemotherapy; } \\
\text { Autologous Stem Cell } \\
\text { Transplant }\end{array}$ & Months & \\
\hline $\begin{array}{l}\text { Deckelbaum et al., } \\
\text { 2014; } \\
\text { Long Beach, } \mathrm{CA}^{12}\end{array}$ & $\begin{array}{c}73 \\
\text { Male }\end{array}$ & Testicular Lymphoma & $\begin{array}{l}\text { Chemotherapy; } \\
\text { Radiation }\end{array}$ & 13 Years & \\
\hline $\begin{array}{l}\text { Mayo et al., 2015; } \\
\text { Birmingham, } \mathrm{AL}^{13}\end{array}$ & $\begin{array}{c}43 \\
\text { Male }\end{array}$ & Mantle Cell Lymphoma & $\begin{array}{l}\text { Chemotherapy; } \\
\text { Autologous Stem Cell } \\
\text { Transplant }\end{array}$ & 4 Years & \\
\hline $\begin{array}{l}\text { Takahashi et al., 2015; } \\
\text { Hokkaido, Japan }{ }^{14}\end{array}$ & $\begin{array}{c}63 \\
\text { Female }\end{array}$ & AML & $\begin{array}{l}\text { Chemotherapy; } \\
\text { Autologous Stem Cell } \\
\text { Transplant }\end{array}$ & 15 Years & \\
\hline $\begin{array}{l}\text { Aung et al., 2017; } \\
\text { Houston, TX }\end{array}$ & $\begin{array}{l}45 \\
\text { Male }\end{array}$ & AML & $\begin{array}{l}\text { Chemotherapy; } \\
\text { Allogeneic Stem Cell } \\
\text { Transplant }\end{array}$ & 3 Months & $\begin{array}{l}\text { Transplant complicated by } \\
\text { CMV infection; } \\
\text { Lesions positive for HPV } \\
\text { and MCPyV }\end{array}$ \\
\hline $\begin{array}{l}\text { Lim et al., 2018; } \\
\text { London, United } \\
\text { Kingdom }^{16}\end{array}$ & $\begin{array}{c}63 \\
\text { Female }\end{array}$ & Breast Cancer & $\begin{array}{l}\text { Chemotherapy; } \\
\text { Radiation }\end{array}$ & 6 Years & \\
\hline $\begin{array}{l}\text { Valdebran et al., } 2018 \\
\text { Irvine, } \mathrm{CA}^{17}\end{array}$ & $\begin{array}{c}32 \\
\text { Female }\end{array}$ & $\begin{array}{l}\text { Acute Promyelocytic } \\
\text { Leukemia (APML) }\end{array}$ & $\begin{array}{l}\text { Chemotherapy; } \\
\text { Bone Marrow } \\
\text { Transplant }\end{array}$ & 7 Years & \\
\hline $\begin{array}{l}\text { Nguyen et al., } 2019 \\
\text { Los Angeles, } \text { CA }^{18}\end{array}$ & $\begin{array}{l}58 \\
\text { Male }\end{array}$ & Large B-Cell Lymphoma & $\begin{array}{l}\text { Chemotherapy; } \\
\text { Radiation }\end{array}$ & 10 Years & \\
\hline $\begin{array}{l}\text { Nguyen et al., } 2019 \\
\text { Los Angeles, } \text { CA }^{18}\end{array}$ & $\begin{array}{c}72 \\
\text { Male }\end{array}$ & $\begin{array}{l}\text { 1) Mantle Cell } \\
\text { Lymphoma } \\
\text { 2) Prostate Cancer }\end{array}$ & $\begin{array}{l}\text { 1) Chemotherapy; } \\
\text { Stem Cell Transplant } \\
\text { 2) Radiation }\end{array}$ & 10 Years & \\
\hline $\begin{array}{l}\text { Marsh et al, } 2020 \\
\text { Columbus, } \mathrm{O}^{19}\end{array}$ & $\begin{array}{c}47 \\
\text { Female }\end{array}$ & AML & $\begin{array}{l}\text { Chemotherapy; } \\
\text { Total Body Radiation; } \\
\text { Allogeneic Stem Cell } \\
\text { Transplant }\end{array}$ & 3 Years & $\begin{array}{l}\text { Transplant complicated by } \\
\text { GVHD }\end{array}$ \\
\hline
\end{tabular}


continued to develop 14 eccrine poromas in several different areas of chronically irradiated skin over the course of 12 years. ${ }^{3}$

Primary malignancies associated with poromatosis were reportedly treated with various combinations of therapy, involving chemotherapy, radiotherapy, or stem cell transplantation. The correlation of poromatosis following the use of chemotherapeutic agents suggests that the accumulation of cytotoxic metabolites potentially alters the structural integrity of the sweat glands, facilitating synchronous occurrence of several eccrine poromas. ${ }^{8}$ After cell damage or trauma occurs, cellular repair is initiated through remodeling and regeneration, creating increased potential for neoplastic transformation. Such changes occur gradually over time, leading to the delayed presentation of lesions. ${ }^{19}$ Similarly, clusters of eccrine poromas have appeared in areas of irradiated skin or within preexisting chronic radiation dermatitis, suggesting $x$-ray damage may also contribute to tumor development. ${ }^{3,20}$

In the initial treatment of the primary malignancies, only 3 previous cases of malignancy-associated poromatosis have involved the use of all 3 therapeutic modalities mentioned above. . $^{75,19}$ In each case, the post-transplantation course was complicated by either graft-versus-host disease or cytomegalovirus infection., 75,19 This finding suggests a possible role of the immune response creating inflammatory damage to the sweat ducts, leading to emergence of eccrine poromatosis. Our patient presents a unique clinical course, as he was treated in a stepwise fashion. He initially received polychemotherapy, followed by radiation after his first relapse, and bone marrow transplant was used as salvage therapy after a second relapse. While multiple etiologies have been suggested, overall, immunosuppressed patients appear to be particularly vulnerable to formation of multiple eccrine poromas. At this time, there is no evidence in the literature to suggest poromatosis is a harbinger of malignancy recurrence or relapse. However, patients with a history of malignancy should continue to be cognizant of signs and symptoms of recurrence as directed by their oncologist.

Conflict of Interest Disclosures: None

Funding: None

Corresponding Author:

Suzanne Alkul, MD

Department of Dermatology

Baylor College of Medicine

1977 Butler Blvd

Houston, TX 77030

Phone: 713-798-6131

Fax: 713-798-6923

Email: ALKUL@bcm.edu

\section{References:}

1. Wankhade V, Singh R, Sadhwani V, Kodate P. Eccrine poroma. Indian Dermatol Online J. 2015;6(4):95-98.

2. Sawaya JL, Khachemoune A. Poroma: a review of eccrine, apocrine, and malignant forms. Int $J$ Dermatol. 2014;53(9):1053-1061.

3. Kurokawa M, Amano M, Miyaguni $\mathrm{H}$, et al. Eccrine poromas in a patient with mycosis fungoides treated with electron beam therapy. Brit J Dermatol. 2001;145(5):830-833.

4. Mahlberg MJ, Mcginnis KS, Draft KS, Fakharzadeh SS. Multiple eccrine poromas in the setting of total body irradiation and immunosuppression. J Am Acad Dermatol. 2006;55(2):S46-S49.

5. Navi, D., Fung, M., \& Lynch, P. J. Poromatosis: the occurrence of multiple eccrine poromas. Dermatol Online J. 2008;14(1).

6. Sherman V, Reed J, Hollywood K, et al. Poromas and porokeratosis in a patient treated for solidorgan and haematological malignancies. Clin Exp Dermatol. 2009;35:e130-e132.

7. Diamantis ML, Richmond HM, Rady PL, et al. Detection of Human Papillomavirus in Multiple Eccrine Poromas in a Patient With Chronic Graftvs-Host Disease and Immunosuppression. Arch Dermatol. 2010;147(1):120-122. 
8. Fujii K, Aochi S, Takeshima C, et al. Eccrine Poromatosis Associated with Polychemotherapy. Acta Derm Venereol. 2012;92(6):687-690.

9. Nguyen BT, Lortscher, DN, \& Lee RA. Multiple poromas in a bone marrow transplant recipient: $A$ case report. Dermatol Online J. 2012;18(7).

10. Miura T, Yamamoto T. Eruptive poromatosis following radiotherapy. Am J Dermatopathol. 2013;35:615-617.

11. Garshick M, DeFilippis EM, Harp J, et al. Eccrine poromatosis in a patient with acute myeloid leukemia following chemotherapy. Dermatol Online J. 2014;20(11).

12. Deckelbaum S, Touloei K, Shitabata PK, Sire DJ, Horowitz D. Eccrine poromatosis: case report and review of the literature. Int J Dermatol. 2013;53(5):543-548.

13. Mayo TT, Kole L, Elewski B. Eccrine Poromatosis: Case Report, Review of the Literature, and Treatment. Skin Appendage Disord. 2015;1(2):95-98.

14. Takahashi C, Nishi K, Minami-Hori M, et al. Multiple poromas following combination chemotherapy and autologous peripheral blood stem cell transplantation. J Dermatol. 2015;42:430-432.

15. Aung PP, Rady PL, Nagarajan P, et al. Detection of Merkel Cell Polyoma Virus and Beta Human Papillomavirus in Multiple Eccrine Poromas in a Patient with Acute Leukemia Treated with Stem Cell Transplant. Am J Dermatopathol. 2017;39(6):489-491.

16. Lim TM, Weir J, Fearfield L. Eruptive poromatosis in a patient with breast cancer. $J$ Cutan Pathol. 2018;45:708-710.

17. Valdebran MA, Hong C, Cha J. Multiple eruptive eccrine poromas associated with chemotherapy and autologous bone marrow transplantation. Indian Dermatol Online J. 2018;9:259-261.

18. Nguyen K, Kim, G, \& Chiu, M. Eccrine poromatosis following chemotherapy and radiation therapy. Dermatol Online J. 2019; 25(11).

19. Marsh RL, Kaffenberger B, Pootrakul L, et al. Multiple Plantar Poromas in a Stem Cell Transplant Patient. Cureus. 2020;12(6): e8773.

20. Ullah K, Pichler E, Fritsch P. Multiple eccrine poromas arising in chronic radiation dermatitis. Acta Derm Venereol. 1989;69(1):70.

21. North JP, Lo J, Landers M. Poromatosis in pregnancy: a case of 8 eruptive poromas in the third trimester. Cutis. 2012;89(2): 81-83.

22. Goldner R. Eccrine Poromatosis. Arch Dermatol. 1970;101(5):606-608.

23. Ogino A. Linear Eccrine Poroma. Arch Dermatol. $1976 ; 112(6): 841-844$.
24. Yoshii Y, Matsuo S, Nishizawa A, Satoh T. Multiple eccrine poromas in a patient with systemic lupus erythematosus. Eur J Dermatol. 2018 Dec $1 ; 28(6): 837$.

25. Wilkinson RD, Schopflocher P, Rozenfeld M. Hidrotic Ectodermal Dysplasia With Diffuse Eccrine Poromatosis. Arch Dermatol. 1977;113(4):472-476.

26. Whitley DB, Mansell JEKL. Multiple eccrine poromas in the paw of a dog. Veterinary Dermatology. 2011;23(2):167-e34.

27. Nishioka M, Kunisada M, Fujiwara N, Oka M, Funasaka Y, Nishigori C. Multiple apocrine poromas: a new case report. $J$ Cutan Pathol. 2015 Nov;42(11):894-896.

28. Hashizume S, Ansai S, Matsuoka Y, Omi T, Kawana S. Case of multiple apocrine poroma in a patient without receiving radiation or chemotherapy. J Dermatol. 2014 Feb;41(2):174175. 